\title{
Development and Evaluation of Talazoparib Nanoemulsion for Systemic Therapy of BRCAI-mutant Cancer
}

\author{
NEELESH KUMAR MEHRA ${ }^{1}$, RAJESHWAR R. TEKMAL ${ }^{2}$ and SRINATH PALAKURTHI ${ }^{1}$ \\ ${ }^{1}$ Department of Pharmaceutical Sciences, Irma Lerma Rangel College of Pharmacy, \\ Texas A\&M Health Science Center, Kingsville, TX, U.S.A.; \\ ${ }^{2}$ Department of Obstetrics and Gynecology, \\ The University of Texas Health Science Center at San Antonio, San Antonio, TX, U.S.A.
}

\begin{abstract}
Aim: This is a debut study report on talazoparib (BMN-673)-loaded nanoemulsion (TZNE) for parenteral administration. Materials and Methods: TZNE (0.05\% drug, $151.4 \pm 0.7 \mathrm{~nm}$ droplet size, polydispersity index of $0.120 \pm 0.010$ and zeta potential of $-33.30 \pm 1.22 \mathrm{mV}$ ) was designed, developed and characterized using in vitro studies. A cumulative in vitro release study was performed in physiological phosphate buffer solution at different $p H$ (5.3, 6.5 and 7.4) using a dialysis method. Cytotoxicity and apoptosis assays were performed on MDA-MB-231, NCI/ADR-RES, 2008 C13, CP-70 and SKOV-3 cell lines using CellTiter ${ }^{\circledR}$ Blue. Quantitative and qualitative cell uptake was studied using fluorescent probe, coumarin-6 (C6). Results: The drug release form TZNE nanoemulsion was slow and sustained for $24 \mathrm{~h}$. Cytotoxicity and apoptosis were found to be concentration-dependent. The half-maximal inhibitory concentration of TZNE was 0.4852 and 1.35, 11.757 and 0.4696 , and 1.169 and $0.7235 \mu M$ in MDA-MB231, SKOV-3 and NCI/ADR-RES cells with 48 and $72 h$ incubation, respectively. Cellular uptake studies using fluorescent probe, coumarin-6 C-6, showed higher cellular uptake of TNZE compared with free C6. Results suggest that nanoemulsion could provide a new platform for systemic delivery of talazoparib.
\end{abstract}

Nanomedicines have gained immense attention in the past few decades because of their efficacy in targeted delivery due to the enhanced permeation and retention effect (EPR), and their ability to overcome chemoresistance in cancer

Correspondence to: Department of Pharmaceutical Sciences, Irma Lerma Rangel College of Pharmacy, Texas A\&M Health Science Center, Kingsville, TX 78363, U.S.A. Tel: +1 3612210748, e-mail: palakurthi@pharmacy.tamhsc.edu

Key Words: Nanoemulsion, talazoparib, cell uptake, breast cancer, $B R C A 1$, cytotoxicity. cells. Nanoemulsions are commonly used vehicles for the delivery of drugs with poor solubility and bioavailability (1, 2). Nanoemulsions are comprised of various excipients such as surfactant, co-surfactant, and oil, and are usually oil-inwater droplets having a mean droplet size ranging from 20 to $200 \mathrm{~nm}$ (3-5). Bu et al. reported self-assembling nanoemulsion using tocopheryl polyethylene glycol 1000 succinate (vitamin E)-loaded with paclitaxel to circumvent drug resistance in breast cancer. The mean diameter of paclitaxel-loaded nanoemulsion was $24.17 \pm 11.24 \mathrm{~nm}$, with entrapment efficiency of $98.94 \pm 0.48 \%$ and drug loading of $1.11 \pm 0.06 \%$ (2).

Poly(ADP-ribose) polymerase (PARP) enzymes are critical for recognition and repair of DNA breaks through the base excision repair pathway and homologous recombination pathway. PARP inhibitors (PARPi) are an exciting and new class of therapeutics for breast cancer 1 gene (BRCA)-related ovarian and breast cancer (6-9). PARPi were shown to be particularly effective in patients with defects in homologous recombination-deficient cancer (10). Because of synthetic lethality of PARPi, they have great clinical potential in therapy of triple-negative breast cancer. PARPi may also be effective against human epidermal growth factor-2 (HER2)- positive breast cancer regardless of defects in the homologous recombination pathway, but not effective in HER 2 knockdown cell lines. It is suggested that PARP is a co-activator of nuclear factor kappa-light-chain-enhancer of activated B cells (NF$\mathrm{kB}$ ), and HER2 expression is linked to NF- $\mathrm{kB}$, therefore, HER2-positive cancers are susceptible to PARP inhibition. Some PARPi exert their cytotoxic activity mainly by suppressing catalytic activity of PARP (veliparib), while other PARPi trap the PARP 1 and PARP 2 enzymes at the damaged DNA (talazoparib, and olaparib) (11).

Olaparib (Lynparza; AstraZeneca, London, UK) is the first PARPi approved by the European Medicines Agency and US Food and Drug Administration (FDA) for responding patients with BRCAl/2-mutant ovarian cancer following platinum chemotherapy, while other potent PARPi, rucaparib 
(AGO14699; Clovis, Boulder, CO, USA), niraparib (MK4827; Tesaro, Waltham, MA, USA), and veliparib (ABT-888; Abbvie, North Chicago, IL, USA) are in late phase clinical trials. Among them, rucaparib was given accelerated approval for use as monotherapy in patients with $B R C A 1 / 2$-mutant (germline or somatic) advanced ovarian cancer (7).

Talazoparib, the most potent PARPi, was shown to be effective against cancer cells carrying mutant $B R C A 1 / 2$ with effective concentration for half-maximal response $\left(\mathrm{EC}_{50}\right)$ of $0.3 \mathrm{nM}$ in MX-1 (epithelial duct carcinoma) and $5 \mathrm{nM}$ in Capan-1 (pancreatic adenocarcinoma) cell lines (12). Clinical study with talazoparib monotherapy showed efficacy in a cohort of 39 patients, with objective response rate of $65 \%$ in patients with ovarian and peritoneal tumors, and $33 \%$ in those with breast cancer, and is well tolerated $(10,13)$. The minimum toxic dose of talazoparib was $1 \mathrm{mg} / \mathrm{day}$, with common adverse events of neutropenia, thrombocytopenia and anemia. A phase III clinical trial using talazoparib monotherapy with primary endpoint of progression-free survival in patients with metastatic breast cancer with BRCA mutation (NCT01945775) is on-going.

Several PARPi have been used in clinical trials, alone or in combination with other chemotherapeutics such as paclitaxel and carboplatin $(10,14-17)$. Talazoparib (BMN673) is a novel, selective, dual-mechanism PARPi that both affects PARP enzyme activity and is reported to trap PARP on DNA, resulting in death of BRCA1/2-mutated cells. Talazoparib has shown proof-of-concept efficacy as a single agent and in combination with other chemotherapeutics in several preclinical studies and is well tolerated in an ongoing phase I/II clinical study $(18,19)$.

Betacaryophyllene (BCP), a major sesquiterpene in Copaiba oil, has been shown to possess anti-inflammatory, neuroprotective, antioxidant, anticancer (20) and antidepressant properties. BCP was reported to potentiate the anticancer activity of paclitaxel by 10 -fold in three different cancer cell lines (NCI/ADR-RES, DLD-1, L929) (21) by enhancing transmembrane transport and thereby increasing intracellular accumulation of paclitaxel. Solubility of BCP, and its antitumor efficacy and safety profile led us to use BCP as a vehicle for talazoparib.

Talazoparib is a poorly soluble drug. Talazoparib is currently used as oral solution (dissolved in $10 \%$ dimethylacetamide/5\% Solutol HS $15 / 85 \%$ phosphatebuffered saline). Oral bioavailability of talazoparib is reported to be approximately $40 \%$. Developing injectable nanoformulations would enhance tumor localization of the drug by EPR effect, leading to improved anticancer efficacy and reduced systemic toxicity. To the best of our knowledge, there is no nanoformulation reported for talazoparib, except an implant for local delivery (22). Thus the aim of our investigation was to develop and characterize a talazoparibloaded nanoemulsion (TZNE) formulation. In this novel formulation, talazoparib dissolved in BCP was used as the oil phase and polysorbate 80 as the emulsifier. Cytotoxicity and apoptosis analysis were performed on MDA-MB-231, NCI/ADR-RES, SKOV-3, 2008 C13 and CP-70 cell lines using CellTiter ${ }^{\circledR}$ Blue. The apoptosis study was performed on $\mathrm{NCl} / \mathrm{ADR}-\mathrm{RES}$ cell line using flow cytometry.

\section{Materials and Methods}

Materials. Talazoparib was purchased from Selleckchem, Houston, TX, USA. CellTiter-Blue ${ }^{\circledR}$ was purchased from Promega, Madison, WI, USA and (-)-trans-caryophyllene $(\beta-\mathrm{CP})$ was purchased from Sigma-Aldrich, St. Louis, MO, USA. Polysorbate 80 and acetonitrile of high-performance liquid chromatography (HPLC) grade were purchased from Fisher-Scientific (Houston, TX, USA). Ultrapure water was obtained from a Nanopure diamond water purification system from Millipore Sigma (Burlington, MA, USA) and was used in all analyses. Regenerated cellulose (RC; $50 \mathrm{kDa}$ ) was purchased from Spectrum Laboratories (Rancho Dominguez, CA, USA). Polyvinylidene fluoride (PVDF) filter with $0.45 \mu \mathrm{m}$ pore size was purchased from VWR International, SugarIand, TX, USA. Amicon regenerated cellulose (RC) ultrafiltration tube with $30 \mathrm{kDa}$ molecular weight cut-off was purchased from Millipore Sigma (Burlington, MA, USA). Phosphate-buffered saline 10X (PBS; pH 5.3, 6.5 and 7.4) and Dulbecco's modified Eagle's medium (DMEM), penicillin, streptomycin, fetal bovine serum (FBS) and trypsin were purchased from Sigma Aldrich. All other chemicals were of pharmaceutical or analytical reagent grade commercially available as used.

Cell lines. Adriamycin-resistant ovarian cancer (NCI/ADR-RES), cisplatin-sensitive ovarian cancer (SKOV-3), cisplatin-resistant ovarian cancer (2008 C13, CP-70) and triple-negative breast cancer (MDA-MB-231) cell lines were obtained from the American Type Culture Collection, Manassas, VA, USA.

Preparation of TZNE. Talazoparib-loaded BCP nanoemulsion (TZNE) was prepared in two steps by PT3100 Polytron highpressure homogenization system (Fisher Scientific, Houston, TX, USA). The aqueous phase was prepared containing $4 \%(\mathrm{w} / \mathrm{v})$ Tween $80,2.2 \%(\mathrm{w} / \mathrm{v})$ glycerin, $0.1 \%(\mathrm{w} / \mathrm{v})$ sorbic acid, $0.05 \%(\mathrm{w} / \mathrm{v})$ sodium acetate, $0.1 \%(\mathrm{w} / \mathrm{v})$ boric acid, and $0.02 \%(\mathrm{w} / \mathrm{v})$ sodium EDTA dissolved in double-deionized water with continuous stirring at $70^{\circ} \mathrm{C}$ and $\mathrm{pH}$ was adjusted with $1.0 \mathrm{M}$ sodium hydroxide. Talazoparib (5 mg) was dissolved in $\beta$-CP $(400 \mathrm{mg})$ and olive oil $(100 \mathrm{mg})$ with sonication until it completely dissolved. The oil phase was added dropwise into the aqueous phase with continuous stirring for up to $1 \mathrm{~h}$ at room temperature at $13,000 \mathrm{rpm}$ to obtain a coarse emulsion. Then the coarse-emulsion was subjected to high-pressure emulsification using Microfluidizer M-110P (Westwood, MA, USA) at 5,000 psi for 10 volume cycles to obtain the final emulsion. The temperature during the homogenization process was kept constant at $30^{\circ} \mathrm{C}$ using an ice-water bath. Blank BCP nanoemulsion (BLNE) was prepared as above except talazoparib was not added.

Globule size, polydispersity index (PDI) and surface charge measurement. Particle size, PDI and zeta potential of the BLNE and TZNE were determined by dynamic light scattering using Zeta PALS zeta potential analyzer ver. 3.57 (Brookhaven Instruments 
Corporation, Holtsville, NY, USA). The mean droplet size and droplet size distribution was measured by taking $10 \mu \mathrm{l}$ of nanoemulsion and dispersing it in $2 \mathrm{ml}$ deionized water at room temperature. All the measurements were made in triplicate $(n=3)$ at $25^{\circ} \mathrm{C}$.

Viscosity measurement. Viscosity of the emulsions was measured by Brookfield DV3T Rheometer (Brookfield Engineering Laboratories, Inc., Middleboro, MA, USA). All measurements were made using the manufacturer's software (NanoDTS, version 6.34). The viscosity (cP) of TZNE was measured at room temperature $\left(25^{\circ} \mathrm{C}\right)$ and body temperature $\left(37^{\circ} \mathrm{C}\right)$ using Brookfield DV3T Rheometer.

Morphology using transmission electron microscopy (TEM). Size and surface morphology of TZNE formulation was characterized by TEM (JEOL JEM-2010 TEM, Peabody, MA, USA) after drying on a carbon-coated copper grid and staining negatively by $1 \%$ phosphotungstic acid by metal shadowing technique.

HPLC analysis. Drug concentration in the analytical samples was estimated using a Shimadzu HPLC system (Columbia, MD, USA), which was equipped with a LC-20AB solvent pump, SIL-20A HT autosampler, CTO-20A column temperature oven and a model SPD$20 \mathrm{~A} \mathrm{UV/vis} \mathrm{detector.} \mathrm{The} \mathrm{chromatographic} \mathrm{separations} \mathrm{were}$ achieved on a Waters Atlantis (Milford, MA, USA) TC-C18 column $(250 \times 4.6 \mathrm{~mm}$ i.d., $5 \mu \mathrm{m}$ particles) at room temperature. An isocratic elution method with the mobile phase consisting of acetonitrile and methanol $(35: 65)$ at a flow rate of $1.0 \mathrm{ml} \mathrm{min}^{-1}$ was used. The absorbance was measured at a wavelength of $227 \mathrm{~nm}$ and the total chromatographic run time was $10 \mathrm{~min}$. Calibration curves were obtained by plotting the peak area versus the drug concentration. Linearity was observed in the concentration range of $15-4,000 \mathrm{ng} / \mathrm{ml}$ in PBS pH $7.4\left(y=16.383 x-378.48 ; \mathrm{R}^{2}=1\right)$.

Determination of drug loading and loading efficiency. A $100 \mu \mathrm{l}$ TZNE sample was passed through Sephadex ${ }^{\text {TM }}$ G-25 M column (PD10; GE Healthcare Bio-Sciences Corp, Piscataway, NJ, USA) after equilibration with $\mathrm{PBS}(\mathrm{pH}$ 7.4) and 20 fractions were collected. The drug concentration was analyzed in each fraction by HPLC. The encapsulation efficiency (\% EE) was calculated using the following formula:

$$
\% \mathrm{EE}=\frac{(\mathrm{Xt}-\mathrm{Xf})}{\mathrm{Xt}} \times 100
$$

Where Xt was the total drug measured by HPLC, Xf was the free drug measured by HPLC.

The drug loading and drug-loading efficiency of TZNE was determined by measuring the free drug content using also Amicon $\mathrm{RC}$ ultrafiltration tube with molecular weight cut-off of $50 \mathrm{kDa}$ (Sigma Aldrich). In brief, $100 \mu \mathrm{l}$ TZNE sample were added into an Amicon RC ultrafiltration tube and centrifuged at $8,000 \mathrm{rpm}$ for $20 \mathrm{~min}$. The filtrate was collected, centrifuged and analyzed by HPLC.

In vitro drug release. In vitro release behavior of talazoparib from the TZNE formulation was performed using a modified dialysis method using RC membrane with molecular weight cut-off of $50 \mathrm{kDa}$ against PBS (at pH 5.3, 6.5 and 7.4) as dialysis medium at $37 \pm 0.5^{\circ} \mathrm{C}$. Five hundred microliters of TNZE was placed into a pre- treated RC dialysis tube which was then hermetically tied using clips and suspended in a glass container with the dialysis medium stirred at $100 \mathrm{rpm}$ on a magnetic stirrer for $24 \mathrm{~h}$. At predetermined time points, samples were collected from the glass container and replenished with same volume of the dialysis medium to maintain the sink condition. Drug concentration in the samples was determined using HPLC.

Cell culture. The cells were cultured in DMEM (Sigma) containing $10 \%$ FBS (Sigma) supplemented with $1 \%$ penicillin-streptomycin mixture (Sigma) incubated for $24 \mathrm{~h}$ for more than $80 \%$ confluence in a humidified atmosphere with $5 \% \mathrm{CO}_{2}$ at $37^{\circ} \mathrm{C}$. The medium was changed two to three times per week.

Cytotoxicity assay. Cytotoxicity of talazoparib and TZNE on NCI/ADR-RES, MDA-MB-231, SKOV-3, cisplatin-resistant 2008 $\mathrm{C} 13$ and $\mathrm{CP}-70$ cell lines was determined using cell CellTiter-Blue ${ }^{\circledR}$ Cell Viability Assay (Promega, Corporation, Madison, WI, USA), while untreated cells were considered as the control. The cells were seeded at $5 \times 10^{3}$ cells/well in 96-well plates overnight in the complete growth medium. After the preincubation period, the medium was removed by aspiration and replaced with fresh medium containing TZNE at a final concentration range of $15 \mathrm{nM}$ to $10 \mu \mathrm{M}$. After 48 and $72 \mathrm{~h}$ incubation, $15 \mu \mathrm{l}$ of CellTiter-Blue ${ }^{\circledR}$ Reagent (Promega Corporation, Madison, WI, USA) was added and cells were incubated for $3 \mathrm{~h}$. The fluorescence intensity in the wells was recorded using a microplate reader (Infinite ${ }^{\circledR}$ M1000 PRO; TECAN, Mannedorf, Switzerland) at 560/590 nm. The cell viability of untreated cells was taken as $100 \%$ cell survival and relative cell viability and the half maximal inhibitory concentration $\left(\mathrm{IC}_{50}\right)$ values were calculated for each cell line.

Tracking of apoptosis. The cells were seeded at $10 \times 10^{4}$ cells/well in 6-well plates and incubated overnight in complete growth medium at $37^{\circ} \mathrm{C}$ with $5 \% \mathrm{CO}_{2}$. The medium was replaced with fresh medium containing TZNE at a final concentration of 10-20 $\mu \mathrm{M}$. After 3 and $6 \mathrm{~h}$ incubation, the medium was removed and the cells were washed three times with PBS, stained using apoptotic cell quantitation kit following the manufacturer's protocol (Sigma Aldrich). Briefly, cells were harvested after treatment by centrifugation and washed three times with cold PBS, and resuspended the cells in PBS ( $\mathrm{pH} 7.4)$ at a density of about $5 \times 10^{4}$ cells $/ \mathrm{ml}$. The cell suspension was aliquoted into flow cytometry tubes at $100 \mu \mathrm{l} /$ tube. A $5 \mu \mathrm{l}$ of annexin $\mathrm{V}$ and 1-2 $\mu \mathrm{l}$ of propidium iodide (PI) working solution was added and cells were incubated for 15-30 min at room temperature in the dark. The cells were examined under fluorescence microscopy and the apoptotic, necrotic and healthy cells were quantitatively determined using fluorescenceactivated cell sorting (FACS; BD Accuri ${ }^{\mathrm{TM}}$ C6 Flow Cytometer; BD Bioscience, San Jose, CA, USA) (23).

Cellular uptake. To allow visualization of the uptake of the prepared nanoemulsion formulation into cells, coumarin-6-loaded nanoemulsion was prepared and cellular uptake was tested by flow cytometry. C6 was used as a marker for TZNE as model drug because $\mathrm{C} 6$ is lipophilic in nature, with $\log \mathrm{P}$ of 6.1 . Fluorescent dyes, including C-6, offer easy and sensitive detection using fluorescence microscopy or with a plate reader (24). Coumarin-6 loaded nanoemulsion was prepared at a concentration of $0.2 \mathrm{mg} / \mathrm{ml}$ and a concentration equivalent to $10 \mu \mathrm{M}$ was used in the cellular 
Table I. Optimized physicochemical parameters of talazoparib-loaded nanoemulsion (TZNE). Values are presented as the mean $\pm S D(n=3)$.

\begin{tabular}{|c|c|c|c|c|c|}
\hline \multirow[t]{2}{*}{ Formulation Code } & \multirow[t]{2}{*}{ Droplet size $(\mathrm{nm})$} & \multirow[t]{2}{*}{ Polydispersity index } & \multirow[t]{2}{*}{ Zeta potential $(\mathrm{mV})$} & \multicolumn{2}{|c|}{ Viscosity (cP) } \\
\hline & & & & $25^{\circ} \mathrm{C}$ & $37^{\circ} \mathrm{C}$ \\
\hline Blank nanoemulsion & $190.6 \pm 0.2$ & $0.159 \pm 0.028$ & $-34.65 \pm 2.09$ & --- & --- \\
\hline TZNE & $\begin{array}{c}151.4 \pm 0.7 \\
D(10)=39.1 \\
D(50)=69.5 \\
D(90)=123.5 \\
D(95)=145.4\end{array}$ & $0.120 \pm 0.010$ & $-33.30 \pm 1.22$ & $1.40 \pm 0.05$ & $1.04 \pm 0.01$ \\
\hline
\end{tabular}

$D(n): n \%$ of the emulsion globules are at or below the designated size.

uptake study. MDA-MB-231 and NCI/ADR-RES cells were seeded at $10 \times 10^{4}$ cells/well in 6 -well plates overnight in complete growth medium at $37^{\circ} \mathrm{C}$ in $5 \% \mathrm{CO}_{2}$ incubator. The complete growth medium containing coumarin-6 and coumarin-6-loaded nanoemuIsion was added to separate wells and incubated for $3 \mathrm{~h}$. After the incubation period, the cells were washed twice with cold PBS (pH 7.4), and the cellular uptake of coumarin-6 labeled nanoemulsion was viewed by fluorescence microscopy (Eclipse; Nikon, Melville, NY, USA) and quantitatively measured by FACS (BD Accuri $^{\mathrm{TM}}$ C6 Flow Cytometer; BD Bioscience). To differentiate the physical surface adsorption of the coumarin 6-loaded nanoemulsion from internalized emulsion globules, cellular uptake was tested at $4^{\circ} \mathrm{C}$ and $37^{\circ} \mathrm{C}$.

Statistical analysis. The results are expressed as mean \pm standard deviation (SD) $(n=3)$ and statistical analysis was performed with Graph Pad Instat Software (Version 3.00; Graph Pad Software, San Diego, CA, USA) by one-way ANOVA. A probability of $p \leq 0.05$ was considered significant.

\section{Results and Discussion}

Nanoemulsions are an important drug carrier system designed for theranostic applications of poorly water-soluble agents. Currently nanoemuIsions are popular cancer drug delivery systems because of their biocompatibility, ability to load poorly soluble drugs, thermodynamic stability and sustained release of the encapsulated drug. The current status of nanoemulsions in cancer therapy has been comprehensively discussed in a review by Sahu et al. (25).

Since the report of the discovery of talazoparib by Wang et al. (18), no efforts have been reported on the development of injectable nanoparticle formulations, except for a sustained release implant for localized therapy of breast cancer (22). To the best of our knowledge, ours is the first report on talazoparib-loaded nanoemulsion intended for parenteral administration. The TZNE was prepared using BCP as the oil phase and Tween 80 as the emulsifier, and sorbic acid as the preservative. Figure 1 schematically depicts preparation of coarse and fine talazoparib-loaded nanoemulsion. BCP is a sesquiterpene obtained from black pepper, hops, cloves and cannabis. $\mathrm{BCP}$ was reported to potentiate the anticancer activity of paclitaxel by 10 -fold in three different cancer cell lines (21) by enhancing transmembrane transport and thereby increasing its intracellular accumulation. It is suggested that BCP accumulates and causes swelling of the membrane, like many cyclic terpenes, and thereby increases the permeability.

The homogenization speed, time, temperature and phasevolume ratio are the critical process parameters in the formulation development of nanoemulsion. TZNE is milky white in appearance, easily flowable and passable through $0.45 \mu \mathrm{M}$ filters made of PVDF and cellulose acetate membrane. Viscosity of TZNE determined by Ostwald viscometer at $25^{\circ} \mathrm{C}$ and $35^{\circ} \mathrm{C}$ was $1.40 \pm 0.05$ and $1.04 \pm 0.01 \mathrm{cP}$, respectively. The physicochemical parameters of TZNE $(0.5 \mathrm{mg} / \mathrm{ml})$ are shown in Table I.

Droplet size distribution is one of the most important physical characterization parameters of the TZNE. The average droplet size (z-average) and droplet size distribution of the prepared nanoemulsions (BLNE and TZNE) were determined using dynamic light scattering (DLS). The low PDI value $(0.159 \pm 0.028)$ of TZNE revealed a narrow droplet size distribution providing good stability of nanoemulsions. The chemical structure of talazoparib and images of the nanoemulsions are shown in Figure 2A and B. Figure 2C shows a TEM image nanoemulsion.

The droplet size and shape in nanoemulsions are affected by electrical charge, thus electrostatic interaction may play a crucial role in determining the physicochemical properties and stability. The nanosize facilitates the extravasation of the nanoemulsion from the vasculature to tumor interstitial space followed by internalization of the emulsion by the tumor cells (26). Coalescence and Ostwald ripening (increase in globule size with time resulting in non-homogeneous dispersion) are two processes which play an important role in the degradation and stability of nanoemulsion. Ostwald ripening is dependent upon the electrical potential of the droplets, while coalescence originates from the collision of two droplets (27). 
Step 1: Preparation of coarse emulsion

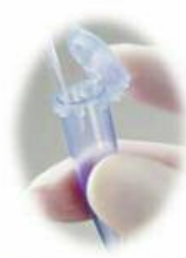

TZP in oil phase

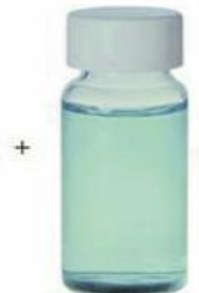

Aqueous phase

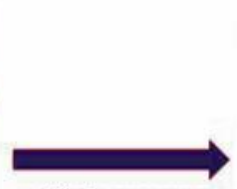

High pressure homogenization

Step 2: Preparation of fine emulsion
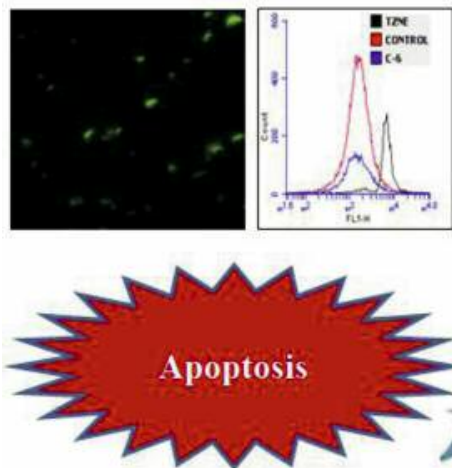

Cell death
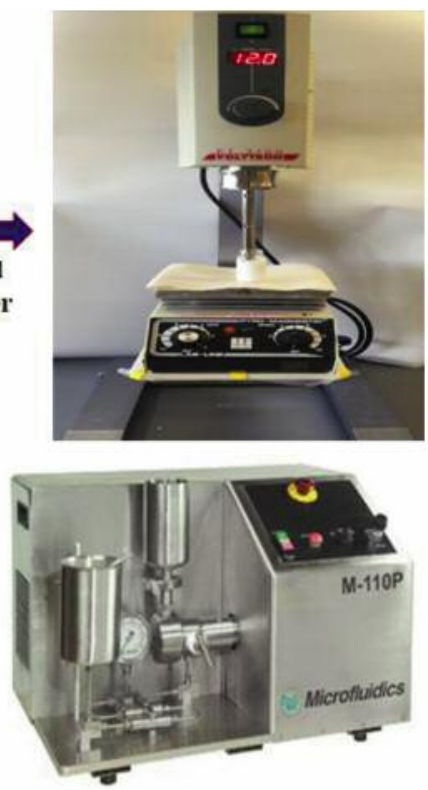

Figure 1. Schematic representation of preparation of nanoemulsions. I: Blank control nanoemulsion. II: Coumarin-6-loaded nanoemulsion (C-6). III: Talazoparib (TZP)-loaded nanoemulsion.<smiles>Cn1ncnc1[C@H]1c2n[nH]c(=O)c3cc(F)cc(c23)N[C@H]1c1ccc(F)cc1</smiles>

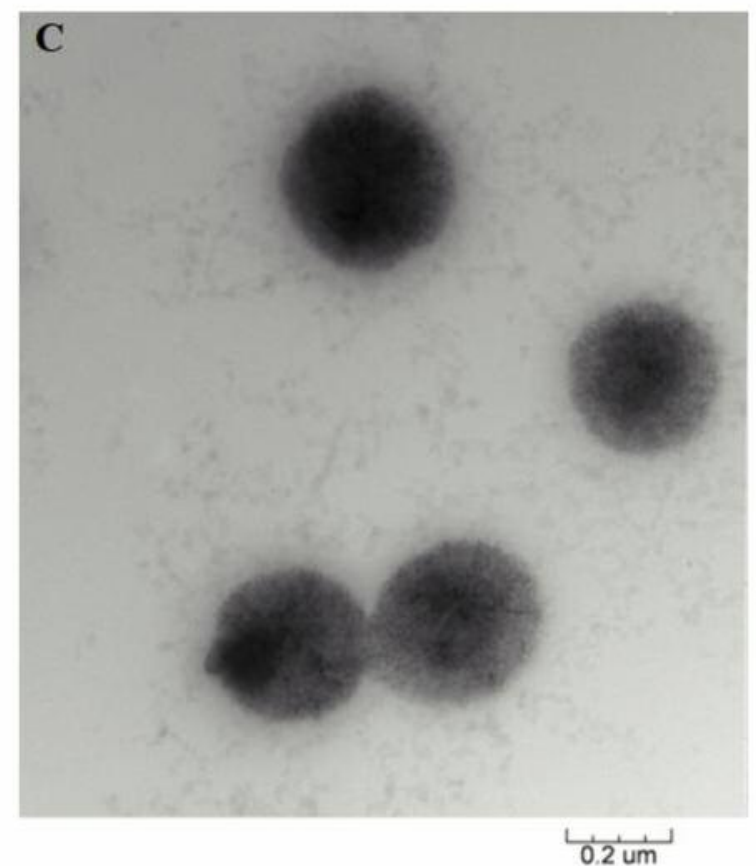

Figure 2. A: Chemical structure of talazoparib. B: Different talazoparib formulations: blank nanoemulsion (I), C-6 loaded nanoemulsion (II), talazoparib-loaded nanoemulsion (III). C: Transmission electron micrograph of talazoparib-loaded nanoemulsion. 

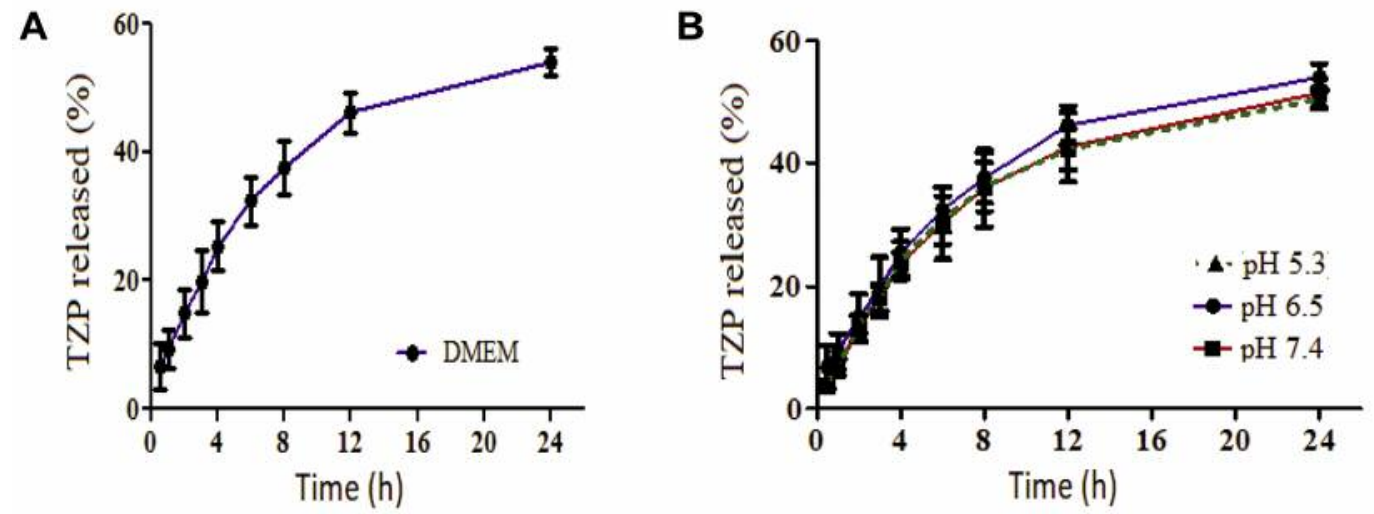

Figure 3. Drug-release study of talazoparib (TZP) nanoemulsion. Cumulative percentage release of TZP in Dulbecco's modified Eagle's medium $(A)$, and phosphate-buffered saline at different $p H(B)$. Values are presented as the mean $\pm S D(n=3)$.
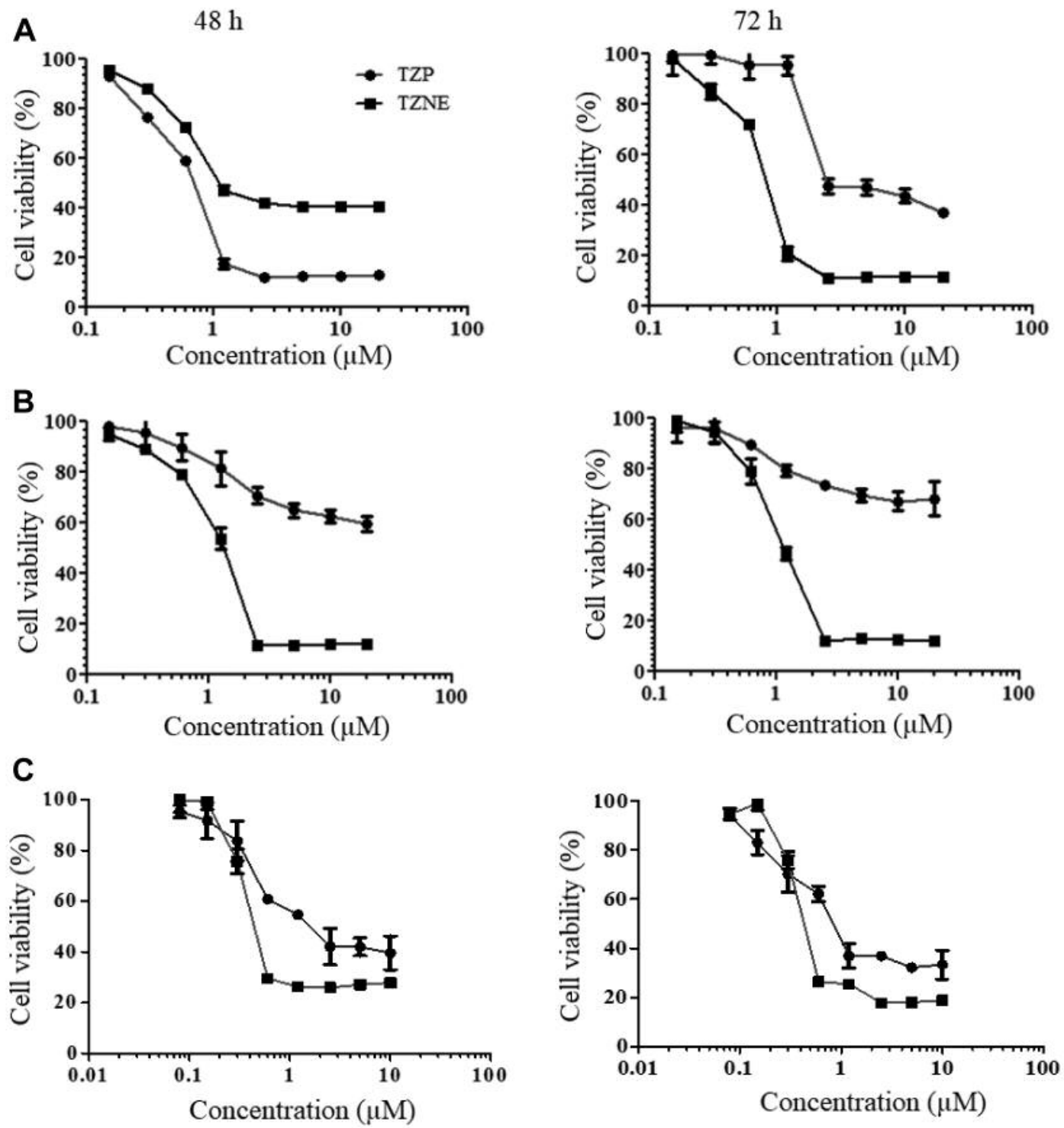

Figure 4. Cytotoxicity of talazoparib (TZP) and talazoparib-loaded nanoemulsion (TZNE) in NCI/ADR-RES (A), SKOV-3 (B) and MDA-MB-231 (C) cell lines using CellTiter-Blue ${ }^{\circledR}$ cell viability assay after $48 \mathrm{~h}$ (left) and $72 \mathrm{~h}$ (right). Values are presented as mean $\pm S D(n=5)$. 

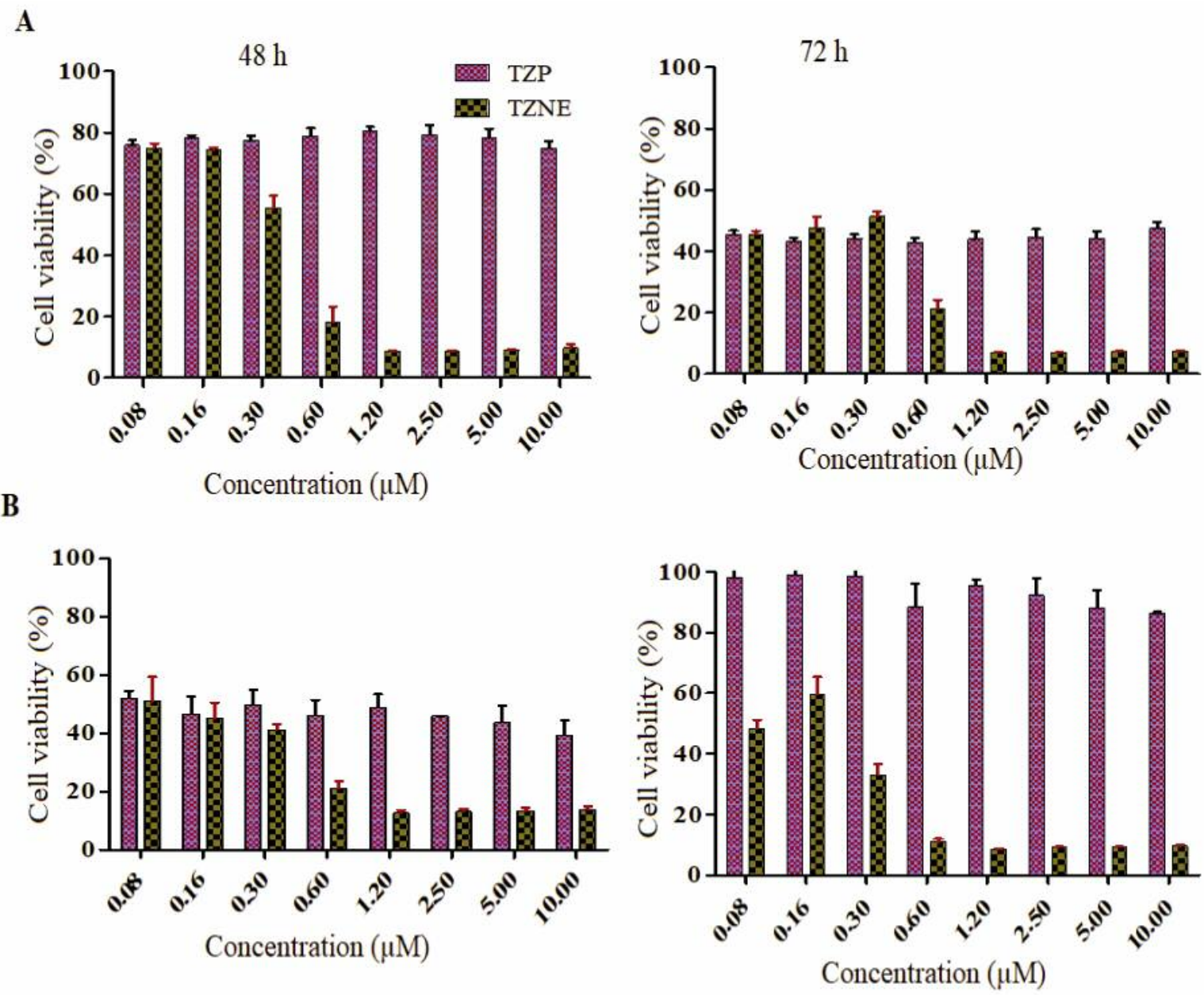

Figure 5. Cytotoxicity of talazoparib (TZP) and talazoparib-loaded nanoemulsion (TZNE) in 2008 C13 (A) and CP-70 (B) cell lines using CellTiterBlue ${ }^{\circledR}$ cell viability assay after $48 \mathrm{~h}$ (left) and $72 \mathrm{~h}$ (right). Values are presented as mean $\pm S D(n=5)$.

Surface charge measurement is an important criterion for nanoemulsion formulation. As the cell membrane is negatively charged, the zeta potential can affect the propensity of the nanoemulsion to interact with the cell membrane, with cationic particles generally displaying stronger interaction thereby eliciting membrane toxicity. The zeta potential represents an electric charge between the shear plane of the final outer (external) layer and the bulk solution, and is mainly influenced by the composition of the nanoemulsion $(28,29)$.

Our group was the first to report a validated quantitative analytical method for talazoparib by HPLC (30). The calibration curve for talazoparib in PBS ( $\mathrm{pH} 7.4$ ) was linear between $15 \mathrm{ng} / \mathrm{ml}$ to $4 \mathrm{mg} / \mathrm{ml}$, with regression equation of $y=16.383 \times-378.48$, and $R^{2}=1$. Gel column (PD10) chromatography was used to determine the drug concentration in the emulsion and the aqueous phase. Talazoparib content in the emulsion phase was found to be $498.92 \pm 0.047(\mu \mathrm{g} / \mathrm{ml})$ and the encapsulation efficiency was more than $99 \%$.
In vitro release drug release is an important quality control test to ensure in vivo product performance and safety. Generally, the tumor extracellular microenvironment is slightly acidic, while the bloodstream $\mathrm{pH}$ is around 7.4. The in vitro release of talazoparib from TZNE was determined using RC $50 \mathrm{kDa}$ dialysis membrane in physiological buffer solution at different $\mathrm{pH}(5.3,6.5$ and 7.4$)$ at $37^{\circ} \mathrm{C}$ as shown in Figure 3. The percentage drug released from TZNE in 24 $\mathrm{h}$ was found to be $50.40 \pm 1.64,47.70 \pm 3.09,51.45 \pm 2.31$, and 54.02 \pm 2.22 in PBS at pH 5.3, 6.5 and 7.4, and DMEM, respectively, indicating the stability and a slow release of the drug from the formulation.

The drug-release data were fit into different mathematical models (zero-order, first order, Higuchi model, HixsonCrowell cube root law and Korsemeyer-Peppas model) to identify the method that best describes the release of talazoparib from the nanoemulsion formulation. The mathematical model that best expressed the kinetic release 


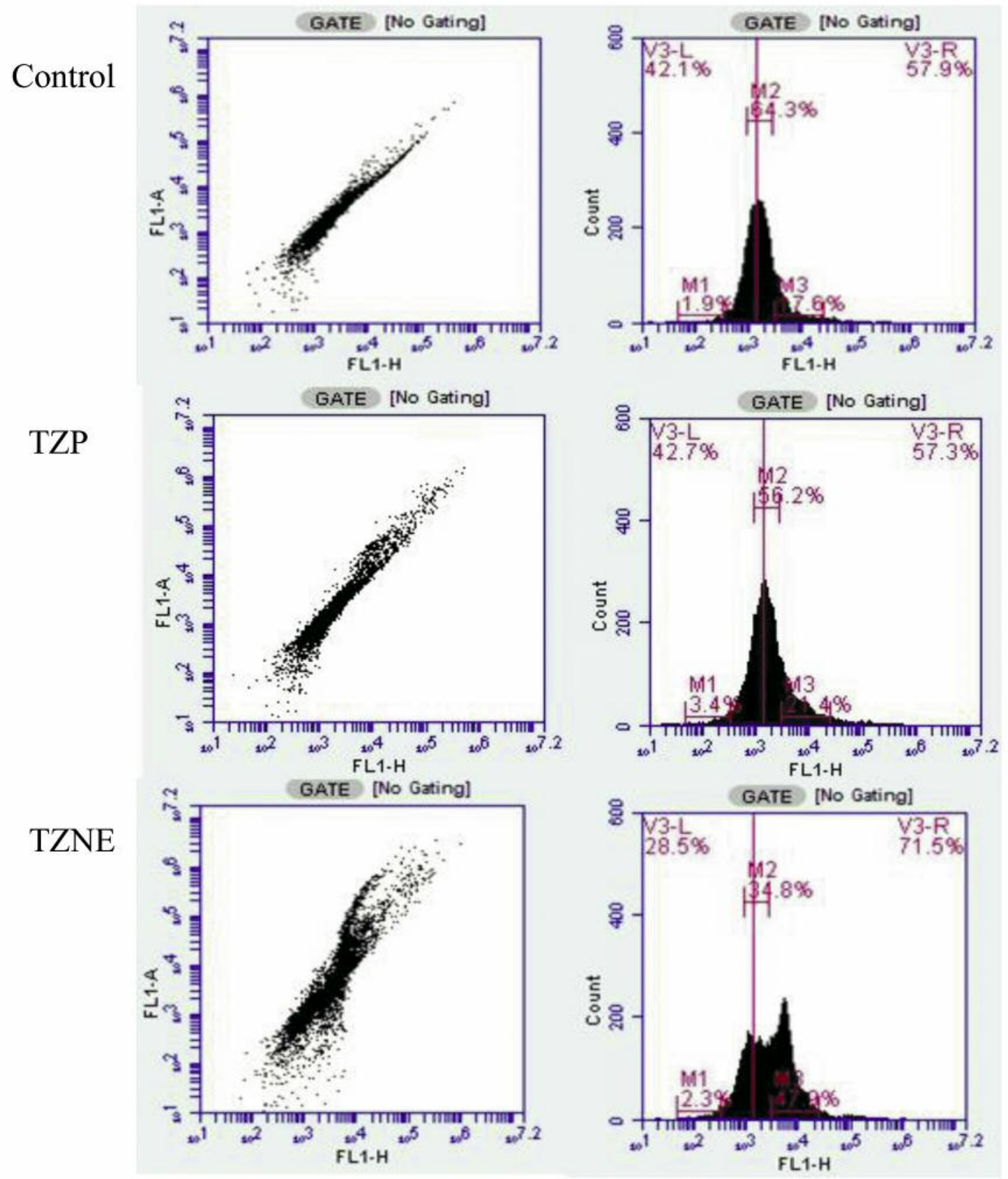

Figure 6. Apoptosis study on the NCI/ADR-RES cell line after treatment with talazoparib (TZP) and talazoparib-loaded nanoemulsion (TZNE) at $10 \mu M$.

profile was selected based on the highest coefficient of

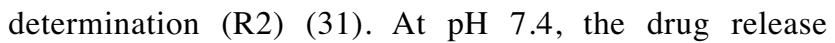
followed first-order kinetics with a slope of 0.033 .

In vitro cytotoxicity of TZNE was assessed in NCI/ADRRES, SKOV-3 and MDA-MB-231 cell lines using CellTiter ${ }^{\circledR}$ Blue cell viability assay at 48 and $72 \mathrm{~h}$. As shown in Figures 4 and 5, talazoparib and TZNE exhibited time-and dose- dependent cytotoxicity. The $\mathrm{IC}_{50}$ value of TZNE was 0.4852 and $1.351,1.757$ and 0.4696 , and 1.169 and $0.7235 \mu \mathrm{M}$ MDA-MB-231, SKOV-3 and NCI/ADR-RES at 48 and $72 \mathrm{~h}$ incubation, respectively. We also assessed cytotoxicity of the emulsion against cisplatin-resistant cell lines, $2008 \mathrm{C} 13$ and CP-70. The $\mathrm{IC}_{50}$ of TZNE against $2008 \mathrm{C} 13$ and $\mathrm{CP}-70$ was 0.30 and $0.12 \mu \mathrm{M}$, and $0.06,0.14 \mu \mathrm{M}$ after 48 and $72 \mathrm{~h}$, 

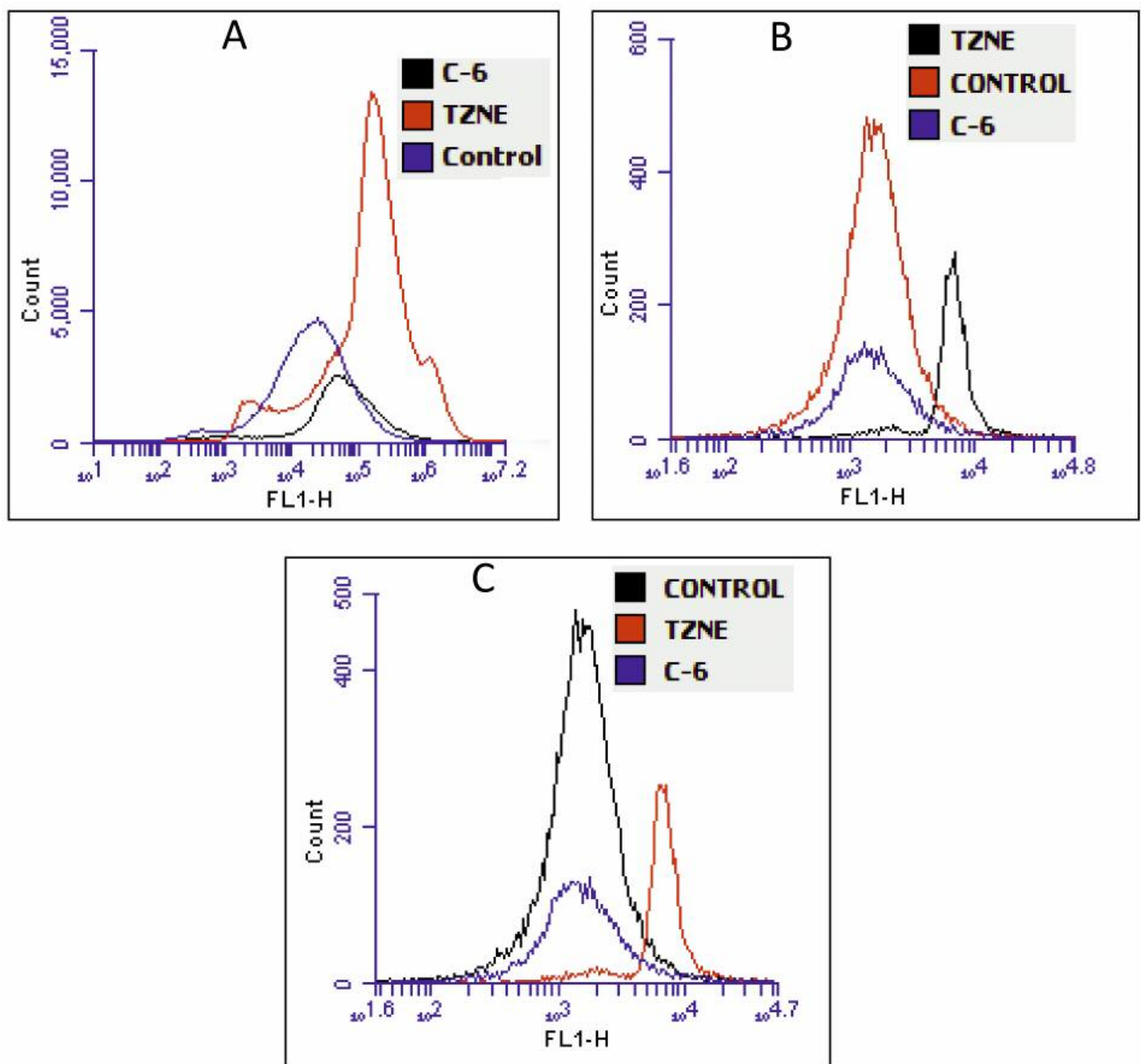

Figure 7. Cellular uptake of coumarin-6-loaded (C-6) and talazoparib-loaded nanoemulsion (TZNE) in NCI/ADR-RES (A), MDA-MB-231 (B) and SKOV-3 (C) cell lines by flow cytometric analysis.

respectively. The IC50 for TZNE was significantly lower against cisplatin-resistant ovarian cancer cells than the sensitive cancer cell lines $(p<0.001)$. These results may be accounted for by the inhibitory effect of BCP on cancer cells, especially of some specific phenotypes. It is known that 200 $8 \mathrm{C} 13$ expresses wild-type $p 53$, while CP70 expresses p53 mutations. It may be speculated that BCP acts synergistically with talazoparib, independently of p53 status $(32,33)$. Unpublished data from our laboratory revealed that BCP was cytotoxic against some cancer cell lines, while it did not affect cell proliferation in others. However, BCP potentiated the cytotoxicity of paclitaxel in the majority of cancer cell lines tested. These results indicate that detailed mechanistic studies in support of potential synergism between BCP and talazoparib are warranted to identify specific cancer phenotypes that are sensitive to the combination therapy. Overall, cytotoxicity of the TZNE formulation was significantly higher than that of free talazoparib in all the cancer cell lines tested at both 48 and $72 \mathrm{~h}$.

Annexin V/PI staining-based apoptosis assay was performed in order to quantitate the different phases of apoptosis in NCI/ADR-RES cell line. Figure 6 shows the results of the 
apoptosis study on NCI/ADR-RES cell line at $10 \mu \mathrm{M}$ talazoparib. A significant increase in the percentage of apoptotic cells was observed when the cells were treated with TZNE as compared to the free drug as well as the blank nanoemulsion $(p<0.05)$. It may be speculated that BCP accumulates in the cell membrane because of its lipophilicity and causes swelling of the membrane, thereby increasing the permeability and cellular accumulation of talazoparib. Increased apoptosis observed with the use of TZNE may be the result of the increase in drug accumulation as compared to use of talazoparib alone.

In this study, the fluorescent dye coumarin 6 was used to facilitate the tracking of BCP nanoemulsion bearing talazoaprib in cancerous cell lines. As shown in Figure 7, cellular uptake was evaluated quantitatively using FACS and qualitatively by fluorescence microscopy. The results of fluorescent microscopy clearly revealed cytoplasmic green fluorescence, owing to internalization of C6-labelled nanoemulsion. Qualitative cellular uptake fluorescence microscopy revealed the higher uptake of C6-nanoemuIsion formulation in cancer cells (data not shown). Results were also confirmed by FACS analysis where fluorescence intensity significantly increased in the case of the C6-nanoemulsion formulation compared to free dye $(p<0.05)$.

\section{Conclusion}

A nanoemulsion formulation was designed and optimized for talazoparib delivery. The in vitro drug-release profiles of TZNE suggest that the drug release was performed in a slow and sustained manner up to $48 \mathrm{~h}$. The high intracellular uptake of TZNE in MDA-MB-231, and NCI/ADR-RES cells may presumably be attributed to the fact that nanoparticles are not a substrate for drug efflux pump unlike the free drug. Cytotoxicity of TZNE was superior to that of the free drug in all cancer cell lines tested in this study. The higher fluorescence intensity clearly revealed the high cellular uptake efficiency of the TZNE formulation. It should be noted that TZNE was formulated with FDA-approved excipients, suggesting that the formulation is expected to be safe and compatible for human use. The present study opens a new door for developing novel formulations for PARPi in general, and talazoparib in particular, potentially increasing their scope of clinical application.

\section{Disclosure}

The Authors declare no conflicts of interest in regard to this work.

\section{Acknowledgements}

This work was supported by the Texas A\&M University College of Pharmacy Research Support to SP.

\section{References}

1 Gehrmann S and Bunjes H: Preparation of lipid nanoemulsions by premix membrane emulsification with disposable materials. Int J Pharm 511(2): 741-744, 2016.

$2 \mathrm{Bu} \mathrm{H}$, He X, Zhang Z, Yin Q, Yu H and Li Y: A TPGSincorporating nanoemulsion of paclitaxel circumvents drug resistance in breast cancer. Int J Pharm 471(1-2): 206-213, 2014.

3 Bouchemal K, Briancon S, Perrier E and Fessi H: Nanoemulsion formulation using spontaneous emulsification: Solvent, oil and surfactant optimisation. Int J Pharm 280(1-2): 241-251, 2004.

4 Huang RF, Wei YJ, Inbaraj BS and Chen BH: Inhibition of colon cancer cell growth by nanoemulsion carrying gold nanoparticles and lycopene. Int J Nanomedicine 10: 2823-2846, 2015.

5 Bali V, Ali M and Ali J: Study of surfactant combinations and development of a novel nanoemulsion for minimising variations in bioavailability of ezetimibe. Colloids Surf B Biointerfaces 76(2): 410-420, 2010.

6 Brown JS, O'Carrigan B, Jackson SP and Yap TA: Targeting DNA repair in cancer: Beyond PARP inhibitors. Cancer Discov 7(1): 20-37, 2017.

7 Brown JS, Kaye SB and Yap TA: Parp inhibitors: The race is on. Br J Cancer 114(7): 713-715, 2016.

8 Jones P, Wilcoxen K, Rowley M and Toniatti C: Niraparib: A poly(ADP-ribose) polymerase (PARP) inhibitor for the treatment of tumors with defective homologous recombination. J Med Chem 58(8): 3302-3314, 2015.

9 Caster JM, Sethi M, Kowalczyk S, Wang E, Tian X, Nabeel Hyder S, Wagner KT, Zhang YA, Kapadia C, Man Au K and Wang AZ: Nanoparticle delivery of chemosensitizers improve chemotherapy efficacy without incurring additional toxicity. Nanoscale 7(6): 2805-2811, 2015.

10 Livraghi L and Garber JE: PARP inhibitors in the management of breast cancer: Current data and future prospects. BMC Med 13: 188, 2015.

11 Murai J, Huang SY, Das BB, Renaud A, Zhang Y, Doroshow JH, Ji J, Takeda S and Pommier Y: Trapping of PARP1 and PARP2 by clinical PARP inhibitors. Cancer Res 72(21): 5588-5599, 2012.

12 Wang YQ, Wang PY, Wang YT, Yang GF, Zhang A and Miao ZH: An update on poly(adp-ribose)polymerase-1 (PARP-1) inhibitors: Opportunities and challenges in cancer therapy. J Med Chem 59(21): 9575-9598, 2016.

13 de Bono J, Ramanathan RK, Mina L, Chugh R, Glaspy J, Rafii S, Kaye S, Sachdev J, Heymach J, Smith DC, Henshaw JW, Herriott A, Patterson M, Curtin NJ, Byers LA and Wainberg ZA: Phase i, dose-escalation, two-part trial of the PARP inhibitor talazoparib in patients with advanced germline BRCA1/2 mutations and selected sporadic cancers. Cancer Discov 7(6): 620-629, 2017.

14 Shi Y, Zhou F, Jiang F, Lu H, Wang J and Cheng C: PARP inhibitor reduces proliferation and increases apoptosis in breast cancer cells. Chin J Cancer Res 26(2): 142-147, 2014.

15 Dent RA, Lindeman GJ, Clemons M, Wildiers H, Chan A, McCarthy NJ, Singer CF, Lowe ES, Watkins CL and Carmichael $\mathrm{J}$ : Phase $\mathrm{i}$ trial of the oral PARP inhibitor olaparib in combination with paclitaxel for first-or second-line treatment of patients with metastatic triple-negative breast cancer. Breast Cancer Res 15(5): R88, 2013. 
16 Clark CC, Weitzel JN and O'Connor TR: Enhancement of synthetic lethality via combinations of ABT-888, A PARP inhibitor, and carboplatin in vitro and in vivo using BRCA1 and BRCA2 isogenic models. Mol Cancer Ther 11(9): 1948-1958, 2012.

17 Pratz KW, Rudek MA, Gojo I, Litzow MR, McDevitt MA, Ji J, Karnitz LM, Herman JG, Kinders RJ, Smith BD, Gore SD, Carraway HE, Showel MM, Gladstone DE, Levis MJ, Tsai HL, Rosner G, Chen A, Kaufmann SH and Karp JE: A phase I study of topotecan, carboplatin and the PARP inhibitor veliparib in acute leukemias, aggressive myeloproliferative neoplasms, and chronic myelomonocytic leukemia. Clin Cancer Res 23(4): 899907, 2017

18 Wang B, Chu D, Feng Y, Shen Y, Aoyagi-Scharber M and Post LE: Discovery and characterization of $(8 \mathrm{~S}, 9 R)-5$-fluoro- 8 - $(4-$ fluorophenyl)-9-(1-methyl-1H-1,2,4-triazol-5-yl)-2,7,8,9tetrahydro-3H-pyrido[4,3,2-de]phthalazin-3-one (BMN 673 talazoparib), a novel, highly potent, and orally efficacious poly(adp-ribose) polymerase-1/2 inhibitor, as an anticancer agent. J Med Chem 59(1): 335-357, 2016.

19 Roche H BJ, Eiermann W, Im Y_H, Martin M, Mina L, Rugo H, Visco F, Zhang C, Lokke N, Lounsbury D and Litton J: A phase 3 study of the oral PARP inhibitor talazoparib (BMN 673) in BRCA mutation subjects with advanced breast cancer (EMBRACA). Ann Oncol 26(Suppl 2): ii16, 2015. https:// doi.org/10.1093/annonc/mdv090.1

20 Jung JI, Kim EJ, Kwon GT, Jung YJ, Park T, Kim Y, Yu R, Choi MS, Chun HS, Kwon SH, Her S, Lee KW and Park JH: Betacaryophyllene potently inhibits solid tumor growth and lymph node metastasis of B16F10 melanoma cells in high-fat dietinduced obese C57BL/6N mice. Carcinogenesis 36(9): 10281039, 2015.

21 Legault J and Pichette A: Potentiating effect of beta-caryophyllene on anticancer activity of alpha-humulene, isocaryophyllene and paclitaxel. J Pharm Pharmacol 59(12): 1643-1647, 2007.

22 Belz JE, Kumar R, Baldwin P, Ojo NC, Leal AS, Royce DB, Zhang D, van de Ven AL, Liby KT and Sridhar S: Sustained release talazoparib implants for localized treatment of BRCA1deficient breast cancer. Theranostics 7(17): 4340-4349, 2017.

23 Mehra NK, Verma AK, Mishra PR and Jain NK: The cancer targeting potential of D-alpha-tocopheryl polyethylene glycol 1000 succinate tethered multi-walled carbon nanotubes. Biomaterials 35(15): 4573-4588, 2014.

24 Pretor S, Bartels J, Lorenz T, Dahl K, Finke JH, Peterat G, Krull R, Al-Halhouli AT, Dietzel A, Buttgenbach S, Behrends S, Reichl S and Muller-Goymann CC: Cellular uptake of coumarin6 under microfluidic conditions into hce-t cells from nanoscale formulations. Mol Pharm 12(1): 34-45, 2015.

25 Sahu P, Das D, Mishra VK, Kashaw V and Kashaw SK: Nanoemulsion: A novel eon in cancer chemotherapy. Mini Rev Med Chem 17(18): 1778-1792, 2017.
26 Gianella A, Jarzyna PA, Mani V, Ramachandran S, Calcagno C, Tang J, Kann B, Dijk WJ, Thijssen VL, Griffioen AW, Storm G, Fayad ZA and Mulder WJ: Multifunctional nanoemulsion platform for imaging-guided therapy evaluated in experimental cancer. ACS Nano 5(6): 4422-4433, 2011.

27 Kaur K, Kumar R, Arpita, Goel S, Uppal S, Bhatia A and Mehta SK: Physiochemical and cytotoxicity study of TPGS-stabilized nanoemulsion designed by ultrasonication method. Ultrason Sonochem 34: 173-182, 2017.

28 Choi AJ KC, Cho YJ, Hwang JK and Kim CT: Characterization of capsaicin-loaded nanoemulsion stabilized with alginate and chitosan by self-assembly. Food Bioprocess Technol 4: 11191126,2011

29 Jiang SP, He SN, Li YL, Feng DL, Lu XY, Du YZ, Yu HY, Hu FQ and Yuan $\mathrm{H}$ : Preparation and characteristics of lipid nanoemulsion formulations loaded with doxorubicin. Int $\mathrm{J}$ Nanomedicine 8: 3141-3150, 2013.

30 Hidau MK, Kolluru S and Palakurthi S: Development and validation of a high-performance liquid chromatography method for the quantification of talazoparib in rat plasma: Application to plasma protein binding studies. Biomed Chromatogr 32(2): 2018. doi: $10.1002 /$ bmc.4046

31 Miastkowska M SE, Ogonowski J, Zielina M and Ludzik A: The kinetic study of isotretinoin release from nanoemulsion. Colloids Surfaces A: Physicochem Eng Aspects 510: 63-68, 2016.

32 Vert A, Castro J, Ribo $M$, Vilanova $M$ and Benito A: Transcriptional profiling of nci/adr-res cells unveils a complex network of signaling pathways and molecular mechanisms of drug resistance. Onco Targets Ther 11: 221-237, 2018.

33 Ikediobi ON, Davies H, Bignell G, Edkins S, Stevens C, O’Meara S, Santarius T, Avis T, Barthorpe S, Brackenbury L, Buck G, Butler A, Clements J, Cole J, Dicks E, Forbes S, Gray $\mathrm{K}$, Halliday $\mathrm{K}$, Harrison $\mathrm{R}$, Hills $\mathrm{K}$, Hinton J, Hunter C, Jenkinson A, Jones D, Kosmidou V, Lugg R, Menzies A, Mironenko T, Parker A, Perry J, Raine K, Richardson D, Shepherd R, Small A, Smith R, Solomon H, Stephens P, Teague J, Tofts C, Varian J, Webb T, West S, Widaa S, Yates A, Reinhold W, Weinstein JN, Stratton MR, Futreal PA and Wooster R: Mutation analysis of 24 known cancer genes in the NCI-60 cell line set. Mol Cancer Ther 5(11): 2606-2612, 2006.
Received April 20, 2018

Revised June 26, 2018

Accepted July 5, 2018 